\title{
Parallels between Confucian Philosophy and Quantum Physics
}

\author{
Sung Jang Chung \\ Morristown-Hamblen Healthcare System, Morristown, USA \\ Email: sung.chung@comcast.net
}

Received 22 April 2014; revised 21 May 2014; accepted 27 May 2014

Copyright (C) 2014 by author and Scientific Research Publishing Inc.

This work is licensed under the Creative Commons Attribution International License (CC BY). http://creativecommons.org/licenses/by/4.0/

c) (i) Open Access

\begin{abstract}
A scientific relationship between the Eastern Confucian philosophy and quantum physics is not clearly known in science and philosophy because of lack of concrete data that would substantially enable us to clearly explain it. Recent findings and discoveries in quantum physics and other relevant science, and the Eastern Confucian philosophy are reviewed in this study. The review of Confucian philosophy, quantum physics and personal experiences of precognitive dreams of the author and others reveal considerable parallels between Confucian philosophy and quantum physics from the ontological perspective.
\end{abstract}

\section{Keywords}

Confucian Philosophy; Quantum Physics; Entanglement; I Ching; Jeong Yeok; Ultron-Logotron Theory; Theory of Everything; Precognitive Dream; Probacent Model

\section{Introduction}

The scientific relationship between the Eastern Confucian philosophy and quantum physics is not clearly known in science and philosophy because of lack of concrete data that would enable us to clearly explain it (Capra, 1999; Penrose, 2007; Penrose, Hameroff, \& Kak, 2011; Hawking, 2001; Bohm, 2006; Laszlo, 2008; Chung, 2012).

\section{The Science of Self, Mind and Body}

The philosophy of self defines the essential qualities that make one person distinct from all others. The self is the agent being the source of consciousness, and responsible for the thoughts of mind and actions of body of an individual, enduring through time.

Teachings in religions concerning their relationship among self, mind and body have been given to humanity 
generally in terms of subjective words. It is taught in religions that the self (spirit) leaves the body at death in the physical world and would be born and continue to live on the Other Side, the spiritual world, implicating ontologically existence of self (spirit) and non-self (non-spirit, world and universe). The philosophy of dualism, self and non-self originates from Renè Decartes. Consequently, scientists and philosophers have been investigating to find objective proofs related to their relationship.

The author had unusual personal experiences that difficult mathematical problems were not possibly solved in his manifest consciousness but could be unexpectedly clearly solved in his subconscious mind. It seems that the subconscious mind continued to reason and perfectly solved the problem and then transferred the solution to his manifest conscious mind (Chung, 2009, 2012). The author published the solution of the math problem in the International Journal of Biomedical Computing that expresses a relationship among exogenous stressor, stress and response in biological phenomena (Chung, 1995). The math problem was a hard differential calculus problem in his research that was aimed to find a general mathematical formula of hazard rate of a general formula, "probacent”-probability equation, that expresses a relationship among intensity of stimulus, duration of exposure and occurrence of response in biological phenomena (Chung, 1960, 2009, 2013b).

The author proposed a theory that there are in human individuals two selves, one, the inner self (the true self) and one, the physical self (the false self) that coexist in one individual person (Chung, 2009, 2012). McGonigal (2012), psychologist at Stanford University, published her book in which she described two minds or two selves in one human individual, naming them "I WILL" and "I WANT" self on the basis of extensive studies on adult subjects. There is a remarkable agreement between the inner and physical selves of Chung and the "I WILL" and "I WANT" selves of McGonigal (Chung, 2012).

Rhawn Joseph (2011c, 2011d) describes presence of two minds in the split-brain of patients with surgical corpus callosotomies. The author postulates that in voluntary movements, the left hemisphere of the brain is dominantly involved in processes of the inner self's control of the physical self that is more associated with the right hemisphere. Consequently, the right half of the body tries to voluntarily control the involuntary behavior of the left half of the body in those patients. Clinical findings in patients with the "split-brain" suggest that if pathways between the prefrontal cortex (PFC) and the secondary motor area are disconnected, it makes the voluntary control of the inner self and bimanual cooperation difficult and so involuntary and/or uncontrollable movements associated with the physical false self/body (brain) against the will of the inner true self would result. Analysis of data on clinical findings in patients described by Joseph seem to reveal a possible agreement between clinical findings in patients and the author's theory that the human individual self is composed of two selves (minds), the inner self and the physical self/body, and that the inner true self controls the physical false self.

Recent researches in neurobiology and neuroscience revealed enormous reciprocal synaptic connections between the prefrontal cortices and other brain structures of limbic system, amygdala, hypothalamus, hippocampus; nucleus accumbens, putamen, thalamus, substantia nigra, etc. (Sapolsky, 2004; Banks et al., 2007; Schultz et al., 2000; Rilling \& Insel, 1999; Herman et al., 2005).

More recent researches in neuroscience using functional magnetic resonance imaging (fMRI) discovered that the prefrontal cortex (PFC) of the human brain performs cognitive control, emotion regulation, moral self-control, control of impulsive behavior and guiding brain activities with future goals and rules (Sapolsky, 2004; Miller \& Cohen, 2001; Greene et al., 2001). Ochsner et al. (2002) discovered that in self-directed emotion control, the prefrontal cortex of the human brain is involved in suppressing activities of the limbic system, amygdala of the critical brain region of emotion and feelings such as fear, anxiety, appetite etc. in their studies using fMRI. The prefrontal cortex receives information from sites throughout the rest of cortex.

The author (Chung, 2012) proposed a theory regarding the scientific relationship among self, mind and body based on the above described findings that a human individual, the self is composed of the inner self (the true self) and the physical self (the false self) that would fade and disappear at death of the body. The inner self has will power, cognition, reason, morality, behavior control, emotion regulation, memory retrieval, supervising the physical self/body that senses through sensory organ systems and responds to the external world, and that is impulsive, behaving for pleasure-desire and instinct for living. The inner self controls the physical self/body by interacting with the prefrontal cortex of the human brain.

\section{The Eastern Confucian Philosophy}

The Confucian philosophy is described in the I Ching, 周易, the Book of Changes (William, 1967) and Jeong 
Yeok, 正易, the Book of Right Change (Chung, 2010). Jeong Yeok, 正易 was written by Kim Hang, 金恒 (1826-1898) who was a great Korean scholar and a master of Confucianism, Buddhism and Taoism. Jeong Yeok is said to complete the I Ching, the oldest Chinese philosophy book. He saw an extraordinary symbol of the coming kingdom of heaven, the later heaven illustrated in Figure 1 after eighteen years of hard study and meditation. There will be 30 days of each month, 360 days of a year without using leap years, and with mild climate throughout the year in the kingdom of heaven, the later heaven that is symbolically represented by Jeong Yeok Eight Trigrams，正易八卦 shown in Figure 1. The spiritual world and the human world will unite in the kingdom of the Creator God. Goodness will be boundless in the kingdom of heaven. He said that he wrote the Word, 言 of the Creator God that was told him, in his book, Jeong Yeok, 正易 (Chung, 2009, 2013a).

In Jeong Yeok, the Creator is named the Non-Ultimate, 无極 (Moogeuk, Wu Chi) and represented with a figure 10. All things of the universe are created by the power of the Creator, the Great Ultimate, 太極 (Taegeuk, Tai Chi) that is represented with a figure 1. The Emperor Ultimate, 皇極 (Hwanggeuk, Huang Chi) that is represented with a figure 5 is between the Non-Ultimate, 10 and the Great Ultimate, 1. 10, 5 and 1 are oneness in Jeong Yeok, implying oneness of the three Ultimates. The author infers that the Hwanggeuk, the Emperor Ultimate represents sons of God, Jesus, Buddha, Confucius, Kim Hang and other enlightened saints. It seems to the author that the Non-Ultimate, the Emperor Ultimate and the Great Ultimate are analogous to the trinity, the Father, the Son and the Holy Ghost in Christianity. It seems to the author that the relationship might be symbolically and mathematically imaginable as follows:

A logarithm with a base of 1 is used.

$\log _{1} 1^{0}=0$

$\log _{1} 1^{1}=1$

$\log _{1} 1^{2}=2$

$\log _{1} 1^{3}=3$

$\log _{1} 1^{5}=5$

$\log _{1} 1^{10}=10$

$\log _{1} 1^{\mathrm{n}}=\mathrm{n}$

$\log _{1} 1^{\infty}=\infty$

$\log _{1} 1^{\infty}=\log _{1} 1=1$

Therefore, $10=5=1$; and also $0=1=\infty$. As David Bohm said, "All is in one; one is in all." The Creator is immanent and transcendent (Chung, 2010). The Creator God is immanent in the deeper, higher place of the human inner self as the super self (Chung, 2009), the Holy Spirit (Christianity) and the Buddha nature (Buddhism).

The Great Ultimate created the Two Forms, 兩儀, Yin and Yang, the two primary basic elementary particles that are represented by a broken line (- -) and a solid line (-). The author names Yin and Yang "yin-ultron", 陰極子 and “yang-ultron”, 陽極子, respectively. All things of the universe are generated by movements of “ultrons”, 極子 (Capra, 1999). Movements of ultrons, 極子 are, join, movement, stillness, advance, retreat, combination, separation, attraction, repulsion, expansion and retraction. The yang-ultron and yin-ultron are analogous to an open string and a closed string of string theory in quantum physics.

Trigrams are composed of different combinations of yin- and yang-ultrons as illustrated in Figure 1. Eight trigams in Figure 1 are all possible combinations of trigrams.

It is postulated that the Conscious Mind of the Creator has power of creating all things of the universe, matter and consciousness; and further is the source of the self of the humanity who are children, parts of God. The deepest part of the inner self that is called "the super self" (Chung, 2009) represents the Great Ultimate in Confucianism and the Holy Spirit in Christianity. Therefore, the inner true self is a co-creator and so has power of generating consciousness (mind) and virtual particles of mental force.

There are principles of the Four Symbols, 四象, the Five Elements, 五行 (earth-soil, metal, water, wood, and fire) that represent a cosmic creative principle; and the Sixty Cycle of combinations of ten kan, 干 heavenly stems and twelve chi, 支 earthly branches (Chung, 2009). The principles of the eight trigrams, and the Five Elements are not described in classic physics and quantum physics.

The sixty kap ja, the sexagenary cycle is made by sixty combinations of ten kan, heavenly stems and twelve chi, earthly branches and used to name and record times, days, months and years.

Hexagrams are composed of different combinations of six same and/or different yin- and yang-ultrons. All possible combinations of ultrons make sixty-four haxagrams that are said to represent all changes in the physical world and human events (Capra, 1999). This principle seems to be analogous to S-matrix theory in quantum 
正易八卦圖

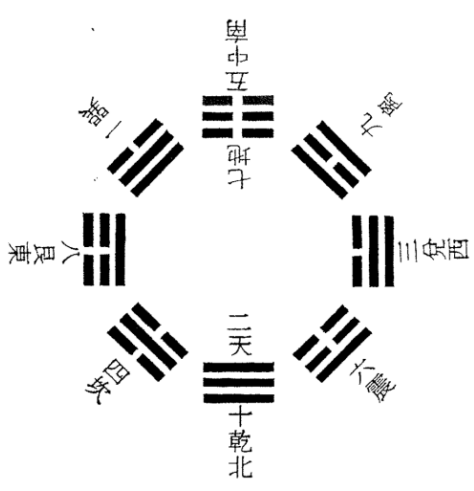

Jeong Yeok Eight Trigrams

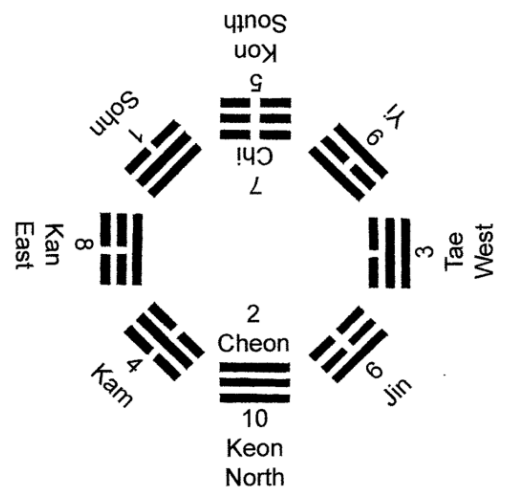

Figure 1. Diagram of the Jeong Yeok Eight Trigrams, 正易八卦. The left diagram is the original diagram and the right diagram is a translated diagram (see text).

physics.

\section{Quantum Physics}

All particles of matter are in essence composed of energy and also can be created from energy. Matter and energy are interchangeable. According to quantum mechanics (Planck, Einstein, Bohr, De Broglie, Heisenberg, Pauli, Schrödinger, Dirac \& Feynman) that describes the physical world on very small atomic and subatomic scales in terms of the laws of quantum physics, a physical change in energy does not occur smoothly and continuously but in discrete steps of minimum unit amount of energy, called quantum. All particles exist in special quantum states.

All particles, electrons, atoms and molecules are in facts waves (Hawking, 1996, 2001; Laszlo, 2008; Suplee, 1999). Quarks and electrons are currently regarded as basic elementary particles. All physical processes in the physical world are based on particles and forces (Hawking, 1996). The force-carrying particle, graviton and photon of bosons are virtual particles. The deeper structure of quarks and electrons is not experimentally observed.

A wave-particle duality of electron proved by Thomas Young and Richard Feynman's double-slit experiment has not been explained (Nichol, 2006). According to David Bohm (2006), this phenomenon suggests that an electron seems to have consciousness, interacting with the environmental background field of space. This is a transformation for a wave to a particle, and vice versa like an organism than interacting parts of an inanimate machine (Nichol, 2006).

Matter, energy and mind are different aspects of the unknown, unbroken flowing movement of totality, the wholeness. Consciousness may be the essential nature of the universe and a more subtle form than matter of the universe (Bohm, 2006). The totality, the wholeness is nonlocal and timeless; it seems to represent the Creator, the Cosmic Consciousness and his activities. Contents of consciousness are called by Bohm "information". The author names “information” "logotron”, 言子 in this study (Greek: logos). The quantum theory of David Bohm seems to be analogous to the teachings in Buddhism that all things of the universe are created by mind, and the universe is in a dust and aeon is in a moment (Dalai Lama, 2005).

Scientific relationships and interactions between self and consciousness: mind and matter in the neurons of the prefrontal cortex and other brain regions are not clearly understood (Gusnard, 2007; Bohm, 2006; Penrose, 2007; Vacariu, 2011; Dresp-Langley \& Durup, 2012) and so need further investigations. Neurons in the PFC seem to play a key role in cognition and the mind-matter relationship (Rational Buddhism, 2012).

All things of the universe, all quantum states of matter may be mathematically determined by the Creator and so could be described with mathematics that is a product of the Creator and the co-creator. However, any quantum states of matter or mathematics cannot create the Creator and/or the co-creator, the self of humanity as the art product cannot create the artist. The relationship between the Creator, the Self or the co-creator, the self of humanity and all created things is analogous to the relationship between the artist and the art products, indicat- 
ing that dualism of Self and non-self is ontologically true in reality and monism, materialist reductionism that self's mind is deducible from non-self as epiphenomenon seems to be not true. The inner true self, spirit independently exists from matter or any quantum states. Biological behavior is primarily controlled and determined by the inner true self, the co-creator in the mind-brain system (Chung, 2009, 2012; Stapp, 1999, 2013).

In string theory of quantum physics, the different types of observed elementary particles are from the different quantum states, string dynamics of strings.

Roger Penrose and Stuart Hameroff (2013) proposed the theory of the orchestrated objective reduction model (Orch OR) with microtubule quantum computations within neurons to explain interactions between mind and matter in the neuron of human brain (Penrose, 2007; Penrose \& Hameroff, 2011). Each of 100 billion neurons of a human brain contains roughly $10^{8}$ protein polymer tubulins that are used for quantum computations correlating with neuronal activities. The Orch OR results in a moment of conscious awareness and/or choice.

Eugene Wigner proposed a hypothesis that consciousness can cause the collapse of the quantum wave function and this determines contents of the brain (Stapp, 2013).

In John von Neumann's theory of emergent dualism, some conscious agent intervenes upon the state of the brain and does conscious choice, controlling the mind-body connection in the brain (Stapp, 1999, 2011). The von Neumann and Heisenberg orthodox quantum theory supports the philosophical dualism in quantum physics. The agent seems to be the inner self of human being.

\section{Precognitive Dreams}

The author had unusual precognitive dreams during the World War II and the Korean War that saved not only the author but also other people. Information received in the precognitive dreams of future events that took place in reality was verified as true (Chung, 2009, 2012, 2014). In the history of the United States, one of the great presidents, Abraham Lincoln had dreams of his death 13 days before he was assassinated (Joseph, 2011). According to Joseph (2011), certain cognitive capacities are well developed in just a few people. Precognitions of future events are well described in religions (the Bible, the Buddhist scriptures).

\section{Quantum Entanglements}

Chien-Shiung Wu and Irving Shaknov (1949) produced positronium that released two photons. David Bohm and Yakiv Aharonov (1957) first recognized quantum entanglement in their analysis of Wu and Shaknov's experiment. John Clauser and Stuart Freedman (1972) produced experimental evidence of entanglement.

Alain Aspect and his collaborators in 1980s, Nicholas Gisin in 1997, and Andrew Watson found in their experiments that the speed of transmission of mutual effect between two entangled particles (e.g., electrons and photons) was faster than the speed of light (Playfair, 2002; Laszlo, 2007; Watson, 1997; Aczel, 2003). The instantaneous influence between two electron twins violates the speed of light according to Einstein's theory of relativity. The phenomenon of entanglement proves that the EPR paradox theory of locality is incorrect and quantum theory of non-locality is correct (John Bell's theorem; Aczel, 2003). The EPR paradox theory of Einstein, Podolsky and Rosen holds the principle of locality that what happens in one local place does not immediately affects what happens in another local place in space. The EPR theory objects to instantaneous fasterthan-light transmission of physical effects between two objects such as photons and electrons at a distance ("spooky action at a distance") that can be observed in entanglement and nonlocality in quantum physics.

Entanglement phenomena are experimentally observed (Aczel, 2003; Penrose, 2007; Penrose, Hameroff, \& Kak, 2011; Laszlo, 2007). Entanglement seems to be explainable by a process of instantaneous influence between two entangled virtual quantum logotrons in consciousness as one of triple properties of the two particles with same quantum states. A change in one particle immediately causes a change in the other particle in nonlocal faster than light communication.

Interactions between self and consciousness: mind and matter are not clearly understood in science and philosophy. There seem to be, to my knowledge, no articles in the literature that clearly explain the relationship between self and consciousness: mind and matter.

\section{Self and Consciousness: Mind and Matter}

The author reviewed modern quantum physics and the Eastern Confucian philosophy. On the basis of the review and authors' personal experiences of valid precognitive dreams, the following theory of "ulron"-"logotron" is 
proposed (Chung, 2014):

1) Valid and undeniable precognitive dreams of the author's personal experience and quantum entanglement strongly suggest the inner self's potential power transcending the time phases of the past, present and future, and existence of a timeless, nonlocal and holographic consciousness world of reality.

2) The "ultrons" are the building blocks of matter of the universe. The "logotrons" are the building blocks of consciousness of human mind. The "logotrons" are virtual particles. The "ultrons" and "logotrons" interact each other with mental-force-carrying "mentalon" in neurons of human brain. Mentalon exchanges between logotron and logotron or logotron of self's consciousness and logotron of consciousness superpositioned to particles of matter.

3) Entanglement could be explained by mental-force-carrying mentalon that exchanges between two entangled virtual quantum logotrons in particles or logotron in conscious mind and logotron in particles of matter at quantum levels.

4) Valid and undeniable precognitive dreams and experimentally accepted entanglement in quatum physics seem to provide concrete human data that might possibly explain a scientific relationship between self and consciousness: mind and matter.

5) There seem to be parallels between the "ultron”-“logotron” theory and quantum physics from the ontological perspective, and a close agreement between the "ultron"-“logotron" theory and the Penrose-Hameroff's Orch OR theory or the von Neumann-Hisenberg's orthodox quantum mechanics that seem to be correct descriptions and applicable to both the inner self and the physical self/body of humanity, respectively on the basis of the human individual self composed of two selves, one, the inner self and one, the physical self.

Scientific relationships between Confucian philosophy and quantum physics are still not clearly understood in science and philosophy. There seem to be, to my knowledge, no articles in the literature that clearly explain the scientific relationship between them from the ontological perspective. In this study, Confucian philosophy and quantum physics are compared on the basis of ontological perspective and an attempt is made to find possible existence of parallels between Confucian philosophy and quantum physics.

1) Philosophical teachings of the Eastern Confucian philosophy that are described in the I Ching, the Book of Changes (William, 1967) and in Jeong Yeok, the Book of Right Change (Kim, 1885; Yi, 1992; Chung, 2009, 2010) are reviewed and compared with quantum physics.

2) Findings and discoveries in modern quantum physics, string theory and other relevant science in the arena of mind and matter are reviewed (Griffiths, 2005; Stapp, 1999, 2013; Penrose, 2007; Penrose, Hameroff, \& Kak, 2011; Tong, 2009; Ochsner et al., 2002; Schwartz et al., 2005; Close, 2011).

3) Review of some of relevant personal experiences of precognitive dreams of the author and others is included in this study.

Comparison of Confucian philosophy and quantum physics is done. Interpretation, theoretical reasoning and imagination are carried out to reasonably explain parallels between Confucian philosophy and quantum physics.

\section{Results}

\section{1. "Ultron", 極子}

Table 1 shows comparison of characteristic aspects of the ultron-logotron theory and quantum physics. Basic elementary particles, ultrons, 極子 would be analogous to strings of string theory. The ultrons are the building blocks of matter of the physical world. It is postulated that the yin- and yang-ultrons were created in the Big Bang like basic elementary particles, quarks and electrons were created (Hawking, 1996). The yang- and yinultrons are positively and negatively charged, respectively. Movements of ultrons in Confucian philosophy are join, movement, stillness, advance, retreat, expansion and contraction. Movements of strings in quantum physics are split, fission, break, pinch, join, spin and oscillation. If the yin-ultron connects its both ends, it would possibly act like the closed string.

Findings in quantum physics are postulated to be applicable to the ultron.

The ultron is postulated to have a property of particle-wave duality.

The electric charge, $\mathrm{e}_{\text {yin }}$ of yin-ultron may be $-1 \mathrm{e}<\mathrm{e}_{\text {yin }}<0$.

The electric charge of yang-ultron, $\mathrm{e}_{\text {yang }}$ may be $0<\mathrm{e}_{\mathrm{yang}}<+1 \mathrm{e}$.

The ultron mass, $\mathrm{m}_{\text {ultron }}$ may be $0<\mathrm{m}_{\text {ultron }}<$ mass of electron.

If external force comes to a region of space, the regional space will be excited and pulsate, and then a hidden 
Table 1. Comparison of characteristic aspects of the "ultron”-"logotron” theory and quantum physics.

\begin{tabular}{|c|c|c|}
\hline Characteristic & The “ultron”-“logotron” & Quantum \\
\hline aspects & theory & physics \\
\hline $\begin{array}{l}\text { Basic elementary } \\
\text { particle }\end{array}$ & Yin- and yang-ultrons & $\begin{array}{l}\text { Quarks and } \\
\text { electrons }\end{array}$ \\
\hline Form & $\begin{array}{l}\text { A solid (-) and a broken } \\
\text { (- -) line }\end{array}$ & $\begin{array}{l}\text { An open }(\sim) \\
\text { and a closed } \\
(0) \text { string }\end{array}$ \\
\hline Movement & $\begin{array}{l}\text { Join, movement, stillness, } \\
\text { advance, retreat, expansion } \\
\text { and contraction }\end{array}$ & $\begin{array}{c}\text { Split, fission, } \\
\text { break, pinch, } \\
\text { join, spin and } \\
\text { oscillation }\end{array}$ \\
\hline Property & $\begin{array}{c}\text { Triple: particle, wave and } \\
\text { consciousness }\end{array}$ & $\begin{array}{c}\text { Triple: } \\
\text { particle, } \\
\text { wave and } \\
\text { consciousness }\end{array}$ \\
\hline Energy & Yes & Yes \\
\hline Force & Yes & Yes \\
\hline
\end{tabular}

vertical pair of particle-antiparticle emerges in it, causing material actualization of real particle and antiparticle, creating matter.

The author infers that when a matter and antimatter collide and disappear, becoming virtual matter and antimatter, consciousness in the excited state in each of the pair would disappear and become a virtual consciousness in the ground state. Consequently, particles of matter seem to have triple property of particle, wave and consciousness (David Bohm, 2006).

If a mental force is presumably created by the self, it will strike the regional space that could trigger and excite virtual consciousness in the ground state to become consciousness in the excited state, creating conscious mind (Chung, 2009, 2014).

Mind and matter are interrelated and interconnected (Capra, 1999; Chung, 2009, 2014). The space is a common working place for the inner self and the physical self of human individuals whose self is composed of the inner self and the physical self/body (Chung, 2009, 2012).

The deeper structure of quarks and electrons is not experimentally observed (Hawking, 1996). They might be probably composed of yin- and yang-ultrons. It seems to the author that the zig and zag particles in the structure of Dirac electron is suggestive of existence of the yin- and yang-ultrons in the deeper structure of electron (Dirac, 2014; Penrose, 2007), and activities of power of the Creator, the Great Ultimate (Chung, 2010).

\section{2. "Logotron", 言子}

Table 2 shows comparison of characteristic aspects of "ultrons" and "logotrons". The author names "information" that is described by David Bohm in his quantum theory "logotron", 言子. Logotrons in this study represent contents of consciousness of mind in humans such as thoughts, feelings, colors, sounds, concepts of all things of the universe that may be expressed by words.

Logotrons are virtual particles, massless, of a triple property of particle, wave and consciousness, possessing force, and having property of spreading as quantum virtual logotron-mentalon (LM) waves as well as remaining at an original, regional point in the nonlocal, holographic space-time with infinite capacities of storage. Virtual quantum logotron-mentalon (LM) wave transmission, if emitted from the logotron is postulated to be instantaneous in the nonlocal holographic space. Mentalon is mental-force-carrying virtual wave and postulated to be inherent to logotrons.

Processes essentially similar to the above described instantaneous transmission of information (logotrons) throughout the nonlocal, four dimensional space-time seem to be explicitly described in the hypothesis of the quantum holographic model (QH) proposed by Edgar Mitchell (Mitchell \& Staretz, 2011). Quantum hologram 
Table 2. Comparison of postulated characteristic aspects of "ultrons" and "logotrons”.

\begin{tabular}{ccc}
\hline Characteristic & Ultrons & Logotrons \\
\hline aspects & & Conscious mind \\
Element of & Physical matter & Conscious (spiritual) \\
Nature & Physical & Created by \\
Creation & Created by the Creator & the Creator and co-creator \\
& Triple: particle, wave and & Triple: particle, wave and \\
Property & consciousness & consciousness \\
& Real and virtual & Virtual \\
State & Graviton, electromagnetic & Mental-force-carrying \\
Interaction between & force, weak and strong & mentalon \\
themselves & nuclear forces & (Instantaneous) \\
\end{tabular}

information, emitted from all objects of the universe is stored without attenuation at the original source that is called the zero potential field (ZPF) and spreads to and absorbed by all other objects of the universe and stored in the nonlocal holographic ZPF without attenuation. QH information is contained in the amplitude, the frequency, the wavelength, the phase relationship of the underlying interference patterns in the electromagnetic waves emitted from the objects. The QH information is picked up by all other objects by resonance that is called phase conjugate adaptive resonance (PCAR). PCAR is fundamental processes for communication, perception cognition and memory, and occurs among all things of the universe, subatomic particles, inanimate and animate things, cells of human bodies, largest structures in the cosmos (the author's note: information is called logotron in the current study. Logotrons of all things and all events of the cosmos of the past, present and future are stored in the Cosmic Consciousness). Everything of the universe is interconnected.

Extrasensory perception (ESP) of telepathy, clairvoyance, remote viewing (RV), and out-of-body experience (OBE) are explainable by quantum holographic (QH) model of Mitchell as well as the ultron-logotron theory. There is experimental evidence for QH theory (Darling, 2005; Radin, 1997; Aczel, 2003; Mitchell \& Staretz, 2011). A considerable agreement seems to be present between Mitchell theory of the QH model and the ultronlogotron theory of the current study.

Bruce Greyson (2011) describes near-death experiences (NDE) occurring under conditions of general anesthesia and/or cardiac arrest resulting in loss of vital function, absence of cardiac output, absence of respiration and absence of brainstem reflexes of three major clinical signs of death. NDE includes a sense of being out of one's physical body, watching events in the operating room, encountering other deceased people etc. NDE indicates continuation of mental function under extreme conditions of non-functioning heart and brain, strongly suggesting the human inner true self existing independently from the brain-body.

In the ultron-logotron theory, ultrons are created from logotrons (information). Virtual particles produced by annihilation of matter are in essence virtual logotrons that are primary creation of the cosmic mind of the Cosmic Consciousness occurring in the timeless and non-local space-time. Consequently, all things of the universe, ultrons of matter are secondary creation from logotrons of Cosmic Mind. The four known forces, gravitational force, electromagnetic force, and weak and strong nuclear forces are postulated to be different manifestations (contact forces) of mental force, mentalon that is inherent to logotrons, suggesting that particles and forces in the physical world are in essence products of logotrons. This inference in the ultron-logotron theory seems to possibly lead to the Theory of Everything (ToE) that has been long sought by physicists, scientists and philosophers. All things are created by mind (Buddha's teaching) and the Word (the Bible, John 1: 1-3).

The empty space is postulated to be not void but filled with invisible and intangible energy and force, virtual ultrons and logotrons (virtual matter and consciousness), further infinite power and love of the Creator God. Virtual logotrons are expressed as Words in religions. It is said in the Bible (John 1: 1-3), "In the beginning was the Word, and the Word was with God, and the Word was God. The same was in the beginning with God. All things were made by him; and without him was not anything made that was made. There seems to be an agreement between the Bible and the ultron-logotron theory. Vedral's view (2010) describes that "the universe starts empty but potentially with a huge amount of information”; “everything in reality is composed of information” 
(the author's note: information is logotron in the current study). The utron and logotron coexist in particles in a superposition state so that particles have triple property of particle, wave and consciousness (Table 1 and Table 2).

The beginning subject of the Jeong Yeok, the Book of Right Change (Kim, 1885) is "The Word of 10, 5 and 1”. In Confucian philosophy, 10 represents the Non-Ultimate, the Creator God, 5 the Emperor Ultimate, (the author's note: the Son of God) and 1 the Great Ultimate (the author's note: the Holy Spirit, the power of God). There seems to be an agreement between the Confucian philosophy and the ultron-logotron theory as well as Christianity in terms of the Word, "logotron".

\subsection{Interactions between Ultron and Logotron}

Figure 2 illustrates Feynman's spacetime diagram of interactions between ultron and logotron in neurons of the prefrontal cortex. Mental-force-carrying virtual particle, "mentalon” exchanges between logotrons; ultron (logotron of consciousness of particles) and logotron of conscious mind), creating resonance-like effects as graviton exchanges between ultrons of matter. The conscious mind of the self of an individual person has power to generate mentalon as well as logotrons with free will and free choice. Mentalon of mental force is virtual force like graviton of gravitational force; it has two states: the ground state and excited state. The author infers that final quantum states result from the incoming neural stimulus in the neurons of the prefrontal cortex of human brain, then OR (objective reduction) occurs (the quantum wave function collapses) when the physical self and the inner self have conscious perception through quantum computations in the microtubule automata (Penrose \& Hameroff, 2011). In the quantum state of the prefrontal cortex of human brain, the quantum wave function collapses when the inner self who is an observer and does conscious cognitive action with free will and free choice, and with attention, efforts, thoughts and planning in the inner self's mind; then logotrons in the exited state are generated from the ground state, generating conscious cognition in the inner self's consciousness (Figure 2(a)) (Stapp, 1999, 2011; Kafatos, Tanzi, \& Chopra, 2011; Schwartz et al., 2005; Ochsner et al., 2002). The self (the true inner self) has power in the conscious mind to choose logotron in the excited state from its ground state, changing the quantum state of the logotron with free will, free choice, attention and intention, guiding actions (Stapp, 1999; Chung, 2012, 2014). Ultron in the excited state is generated by mentalon originated from the self's conscious mind (Figure 2(b)) (Schwartz et al., 2005; Ochsner et al., 2002). Figure 2(b) illustrates processes at the interface between mind/consciousness and brain. Existence of mentalons can be empirically recognized by their effects in the external world resulting from interactions between mind and matter in the PFC of human brain, for instance, in physical exercises and voluntary movements of hands and extremities. In this matter-mind process, mentalons participate in generation of conscious mind (Figure 2(a)) and vice versa (Figure 2(b)). M line shows its origin on the left side with an apparent slightly upward right direction but is actually horizontal and parallel to the space axis, indicating an instantaneous speed in space-time (Figure 2(a) and Figure 2(b)).

In the ultron-logotron theory, interactions between logotrons are postulated to be triggered and excited by mental-force-carrying mentalon created by conscious mind that exchanges between logotrons, probably accompanying quantum waves of logotrons in superposition. Mentalon is postulated to be able to be created by the inner self's conscious mind without any information (logotrons) in consciousness like in deep meditation with free will and free choice. Mentalon is postulated to be inherent to logotrons as graviton to matter. Mentalon and logotron are probably transmitted together in superposition states. Their exact forms are not known but possibly LM wave form. The Penrose-Hameroff Orch OR theory seems to explain the primary quantum phenomena occurring in neurons of the PFC and other brain regions of human brain in general that result in perception in the physical self's consciousness in the mind-brain system. The von Neumann-Heisenberg orthodox quantum theory is postulated to explain the secondary quantum phenomena superpositioning to the primary quantum phenomena occurring in neurons of the human prefrontal cortex (PFC) that result in cognition, control, choice and memory retrieval in the inner self's conscious mind. Consequently, both theories seem to be correct descriptions of the human mind-brain system.

\section{Discussion}

Results shown in Table 1 seem to reveal parallels between the ultron-logotron theory and quantum physics, and to be supported by findings and discoveries in quantum physics.

Figure 2 suggests that "a self-observing quantum system" is needed to explain the mind-brain system as 


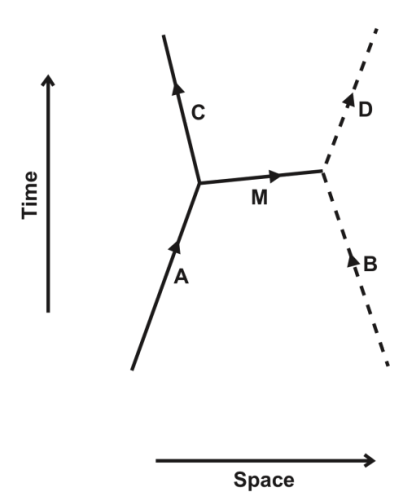

(a)

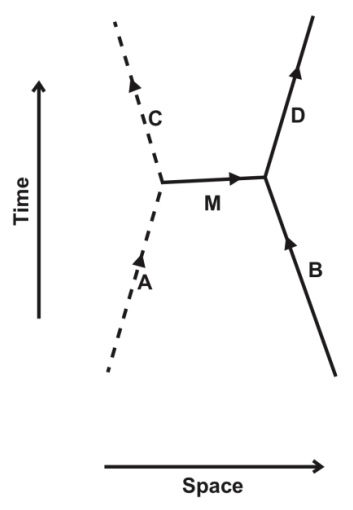

(b)

Figure 2. Feynman's spacetime diagram of interactions between "ultron” and "logotron”. (a) A-Real ultron in the excited state in the neuron of the prefrontal cortex caused by incoming stimulus. C-Real ultron in the ground state after collapse of the quantum wave function when the self controls (observes). B-Virtual logotron in the ground state in the neuron of the prefrontal cortex. D-Virtual logotron in the excited state when the self observes and cognizes. M-Mental-force-carrying "mentalon" exchanging between ultron and logotron; (b) A-Virtual logotron in the excited state in the self's conscious mind in the neuron of the prefrontal cortex. C-Virtual logotron in the ground state after exchanging mentalon between the logotron and ultron in the neuron. B-Real ultron in the ground state in the neuron of the prefrontal cortex. D-Real ultron in the excited state in the neuron after exchanging mentalon between logotron and ultron. $\mathrm{M}-$ mentalon (see text).

Henry Stapp $(1999,2011)$ describes. The self (the human agent) is the observer, the self's mind is analogous to the observing instrument, and the quantum state in the prefrontal cortex is the observee. These three aspects are in the prefrontal cortex of the human mind-brain system. It seems to the author that there is a considerable agreement between the ultron-logotron theory and the von Newmann-Heisenberg's orthodox quantum mechanics (Stapp, 1999, 2011). Jeffrey Schwartz $(1999,2005)$, psychiatrist, reported his successful clinical treatment of patients with obsessive compulsive disorder (OCD) with training to do their own willful redirection of his attention away from intense urge associated with pathological activity in the basal ganglia, strongly suggesting that thoughts of patients can change pathologic states of the brain, and further that the self as the observer, the self's mind analogous to the measuring instrument, and the state of the brain as the observee constitute a unified mind-brain system.

All ultrons and logotrons are postulated to be created in the Big Bang like basic elementary particles in quantum physics (Hawking, 1996, 2001). The ultrons are the building blocks of matter of the physical world; the logotrons are the building blocks of consciousness. The logotrons are not experimentally observed but their existence is understood by their effects and their direct cognition in the self's consciousness, self-awareness.

Table 3 shows comparison of maximum numbers of shell electrons in seven orbital shells, K-Q of atoms that are reported, and formula-derived numbers in quantum physics or in the current ultron-logotron theory. The formula expressing those numbers of electrons in quantum physics is $2 n^{2} ; n=1,2,3,4,4,3$ and 2 in the seven shells. The formula of the ultron-logotron theory is $n \pm 2 ; n=0,10,20,30,30,20$ and 10 in the seven shells. There is a complete agreement between those numbers (Wikipedia, 2013b).

In Confucian philosophy, finger-counting is used in operation of ten kan and twelve chi (stem and branch) of the Sixty Cycle. In continued counting 1 to 32, the left hand with the bent thumb and index finger, and the extended middle, ring and little fingers indicates 2, 8, 18 and 32; then if reverse counting from 32 to 8 is done, the same left hand with the two bent fingers will indicate 32, 18 and 8 . The author may call this regarding to maximum numbers of orbital electrons "a two fingers rule". It reminds the author of Fleming's hand rule illustrating electromagnetic force.

The self can create mental force in conscious mind. Mental force is carried by virtual particle-wave, mentalons between ultron and logotron or between logotrons. Mental force seems to be the fifth force that may be added to the four known forces, gravitational force, electromagnetic force, weak and strong nuclear forces of the physical world.

Stable states in matter suggested in Confucian philosophy are as follows:

1) Integer-3-suggested stable states of basic elementary particles are baryons of six flavors composed of three 
Table 3. Comparison of maximum numbers (N) of shell electrons of orbital shells in atoms in reported (Wikipedia, 2013b), quantum physics and the "ultron”-“logotron” theory.

\begin{tabular}{|c|c|c|c|c|c|c|c|c|}
\hline Orbital shell & & K & $\mathrm{L}$ & M & $\mathrm{N}$ & $\mathrm{O}$ & $\mathrm{P}$ & Q \\
\hline $\begin{array}{l}\text { Maximum } \\
\text { number of } \\
\text { electron (N) } \\
\text { reported }\end{array}$ & & 2 & 8 & 18 & 32 & 32 & 18 & 8 \\
\hline Quantum & $\mathrm{N}=2 \mathrm{n}^{2}$ & 2 & 8 & 18 & 32 & 32 & 18 & 8 \\
\hline physics & $\mathrm{n}$ & 1 & 2 & 3 & 4 & 4 & 3 & 2 \\
\hline The "ultron"- & $\mathrm{N}=\mathrm{n}+$ or -2 & 2 & 8 & 18 & 32 & 32 & 18 & 8 \\
\hline $\begin{array}{l}\text { "logotron" } \\
\text { theory }\end{array}$ & $\mathrm{n}$ & 0 & 10 & 20 & 30 & 30 & 20 & 10 \\
\hline
\end{tabular}

quarks, including two baryons of proton and neutron. Protons and neutrons are composed of three quarks and very stable so that they are constituents of nuclei of atoms and molecules. Natural decay of these two baryons has not been observed (Hawking, 1996). Three quarks in baryons are analogous to trigrams consisting of three ultrons in Confucian philosophy (Figure 1).

All possible combinations of three are eight. All possible trigrams composed of three yin- and yang-ultrons are eight trigrams as shown in Figure 1 in Confucian philosophy. It seems to the author to suggest that existence of eight flavors of quarks, and that two more undiscovered flavors, "light" and "dark" (the author's naming) besides the other six known flavors, up, down, strange, charm, top and bottom, probably one positively and one negatively charged, of undetermined mass, and probably extremely short-lived or virtual quarks like $\mathrm{W}$ and $\mathrm{Z}$ bosons (Close, 2011).

2) Integer-3-suggested amino acids: each of the known 20 amino acids is determined by a series of "triplet code" of three messenger RNA (mRNA) as a copy of chromosomal DNA that specifies its sequence of amino acid of protein (Smith, 2008). The triplet code is analogous to trigrams.

3) The integer-64-suggested chromosomal genetic code consisting of 64 codons that determine production of known amino acids, specifying their sequence by mediation of mRNA in the ribosome as a copy of DNA in the genetic code, and synthesizing all proteins in organic chemistry. There are only four nucleotides (bases) in mRNA: adenine (A), uracil (U), guanine (G) and cytosine (C) in mRNA (Smith, 2008; Carey, 1998; Wikipedia, 2014). The 64 codons in the genetic code seem to be analogous to the sixty-four hexagrams. The four nucleotides, base, A, U, G and C in mRNA are analogous to the Four Symbols in Confucian philosophy. All possible three combinations of four nucleotides $(A, U, G$ and $C)$ are $64\left(4^{3}=64\right)$.

4) The sexagesimal cycle of sixty seconds making one minute, sixty minutes one hour, thirty days (60/2) a month, twelve months $(60 / 5)$ a year and 360 days $(60 \times 6)$ a year (the author's note: in the later heaven), implying that the figure 60 in cycles seems to be analogous to the cyclic sixty kap ja, the sexagenary cycle in Confucian philosophy.

5) Computer science is based on a binary system of two digit values, 0 and 1 . Any integer numbers can be represented by $2^{\mathrm{n}}$ : With two bits $\left(2^{2}\right)$, four numbers of messages can be expressed: $00,01,10$ and 11 ; with three bits $\left(2^{3}\right)$, likewise eight numbers; with six bits $\left(2^{6}\right), 64$ numbers of messages. The yin- and yang-ultrons system seems to be analogous to the binary system of 0 and $1\left(2^{\mathrm{n}}\right)$; with three bits corresponding to the Eight Trigrams, with six bits to the Sixty-Four Hexagrams.

6) Serum cholesterol levels in adults annually increase from young age until 60 years of age, then, the level begins to gradually decrease in man (Chung, 1990, 1992), suggesting 120 years of human life span $(60 \times 2)$.

The author postulates that mathematical equations and principles in quantum physics such as Schrödinger's wave function, Dirac equation, Heisenberg's uncertainty principle, Planck’s formula, Pauli's exclusion principle etc. are applicable to the ultron-logotron theory although Confucian philosophy lacks mathematical descriptions.

However, many macroscopic phenomena such as gender of male and female, day and night, sleep and wakefulness etc. are analogous to the Two Forms, Yin and Yang; microscopic subatomic phenomena such as extremely stable baryons, protons and neutrons that produce atoms and molecules as nuclear components of all things of matter of the cosmos are composed of three quarks, appearing analogous to stable trigrams; the genetic code in living cells that consists of 64 codons is analogous to stable 64 hexagrams; the sexagesimal cycle of 
times, days, months, and years seem to be analogous to the sexagenary cyle of the sixty kan chi; and the binary system of computer science seems to be analogous to the yin- and yang-ultrons in Confucian philosophy; serum cholesterol levels in human adults gradually increase until the age of 60 years, then, begin to decrease. All these phenomena appear to suggest validity of Confucian philosophy. Intuition, enlightenment, revelation and theoretical intuitive reasoning in philosophy and religion seem to be so important in discovering truth like theoretical physics being important in quantum physics.

Current theory in the human evolution holds that the modern human, Homo sapiens migrated from Africa to the Middle East about 120,000 years ago (The National Geography Society, 2008; Joseph, 2001, 2011). According to Jeong Yeok, the year 1884 A.D. was 118,643 year (Chung, 2010, 2012). It seems to the author that the human spirit (the inner true self) probably began to first transmigrate to anthropoid ape-like man, Homo sapiens neanderthalis (incarnate) from the spiritual world to the physical world, then a new era of humanity, modern Homo sapiens sapiens started in the human history of evolution.

The author had a special experience on Mount Kyeryong in Korea where Kim Hang, the author of Jeong Yeok, the Book of Right Change studied all of the Confucian scriptures and ancient Chinese classic books. He achieved enlightenment after eighteen years of hard study and meditation at the age of fifty-four years in 1879 .

One day when I was young, I went to the Mount Kyeryong with one of my friends, Dr. Chong Chul Yook, a physicist, and stayed at a house owned by Cheol Hwa Song to study Jeong Yeok. The residence was used for disciples of Kim Hang. Dr. Jeong Ho Yi who was a professor of philosophy at a university and later became president of the Choongnam National University, and a leading Jeong Yeok and I Ching scholar in Korea was among the scores of disciples there. We spent most of the time doing meditation, prayer and mantras day and night.

The landlord, Cheol Hwas Song was a middle-aged, decent, and extraordinary teacher and also an amazing spirit medium. When he entered his trance and began to speak, we disciples silently listened to his fluent, eloquent, fascinating, sometimes poetic and beautiful speech. We were astonished and overwhelmed with wonder, joy, and faith.

We realized who was speaking from the Other Side because they identified themselves by their names: Chyonjo Sang Jye, the Creator God; Jesus Christ; Confucius; Kim Hang; and Chung Po'eun (the author's ancestor) separately appeared and delivered lectures, giving wonderful teachings in ethics, morality, religion, and philosophy, most emphatically in love and compassion.

Tears were flowing down my cheeks, and my heart was pounding with respect, awe, gratitude, joy and faith.

God said, "A miserable-looking, hungry beggar clothed in rags is standing at the gate in a rainy day. I am within him." God taught us to respect and love our neighbors. The following teachings were also given by other saints.

"Before those who govern the people perform a religious service, look around the country to see if anyone starves. Make sure that no one is starving." "Those who govern the people, let people live in lofty buildings and large houses.”

"Keep your mind as broad as the universe." One of speeches given by Chung Po'eun through the medium, Song in the lecture included that the author is his $19^{\text {th }}$ descendent. This true genealogy of the author was not previously known to Song. Chung Mong Chu (Po'eun, 1337-1392) was a great scholar in Confucianism and a righteous and loyal statesman. He served and died for his king and his country (Chung, 2009).

Christianity and Confucianism as well as other major religions of the world, teach the same thing, love and righteousness; they are multiple paths that lead us up to the mountaintop of the Creator God.

The scientific relationship between Confucian philosophy and quantum physics would need further research.

\section{Conclusion}

Results of this study based on the ultron-logotron theory (Chung, 2014) and review of quantum physics reveal remarkable parallels between Confucian philosophy and quantum physics as follows:

1) Confucian philosophy presents the "ultrons" as the building blocks of the physical world, and the ultron-logotron theory proposes the "logotrons" as the building blocks of the consciousness world at quantum levels.

2) There seem to be remarkable parallels between Confucian philosophy of the Eastern philosophy and quantum physics of the Western science from the ontological perspective. 
3) Results of this study may suggest existence of eight flavors of quarks and that there would be possibly two more undiscovered flavors of quarks, "light” and "dark" (the author's naming) besides the other six known flavors, probably one positively and one negatively charged, of undetermined mass, and probably extremely shortlived or virtual quarks like $\mathrm{W}$ and $\mathrm{Z}$ bosons.

4) There could be primary basic elementary particles, yin- and yang-“ultrons" in the deeper structure of quarks and electrons.

5) The physical world, the consciousness world and the spiritual world of our universe coexist in superposition and represent the macro-cosmos of the Creator God. The superpositioned worlds, the macro-cosmos of the Creator of our universe, the Self, seem to be analogous to the physical body, the conscious mind and the inner spiritual self in superposition that represent the micro-cosmos of the co-creator, the self of the humanity.

6) The spiritual world is invisible but real. The spiritual world and the human world would unite in the coming kingdom of heaven on Earth where goodness will be boundless according to Jeong Yeok, the Book of Right Change of Confucian philosophy as the Bible predicts it.

7) Virtual particles are in essence virtual logotrons (information) that are the archetype of real particles. Annihilation and creation of ultrons (matter) are secondary to action of underlying virtual logotrons in the Cosmic Consciousness (mind). This seems to suggest that the ultron-logotron theory possibly leads to the Theory of Everything (ToE).

Further research would be needed for verification of parallel relationships between Confucian philosophy and quantum physics.

\section{Acknowledgements}

The author thanks the Republic of Korea for the financial assistance in his research (No. 166920).

\section{References}

Aczel, A. D. (2003). Entanglement. New York: Plume.

Banks, A. J., Eddy, K. E, Angstadt, M., Nathan, O. J., \& Phan, K. A. (2007). Amygdala-Frontal connectivity during emotion regulation. Social Cognitive and Attentive Neuroscience, 2, 303-312. http://dx.doi.org/10.1093/scan/nsm029

Bohm, D. (2006). Wholeness and the Implicate Order. New York: Routledge.

Capra, F. (1999). The Tao of Physics. Boston, MA: Shambhala.

Carey, G. (1998). Chapter 3. DNA and the Genetic Code.

http://psych.colorado.edu/ carey/hgss/hgsschapters/hgss_chapter03.pdf

Chung, S. J. (1960). Studies on a Mathematical Relationship between Stress and Reponse in Biological Phenomena. Journal of the National Academy of Sciences, Republic of Korea, 2, 115-162.

Chung, S. J. (1990). Formulas Predicting the Percentile of Serum Cholesterol Levels by Age in Adults. Archives of Pathology and Laboratory Medicine, 114, 869-875.

Chung, S. J. (1992). Relationship among Age, Serum Cholesterol Level and Population Percentile in Adults. International Journal of Biomedical Computing, 31, 99-16. http://dx.doi.org/10.1016/0020-7101(92)90066-2

Chung, S. J. (1995). Formulas Expressing Life Expectancy, Survival Probability and Death Rate in Life Table at Various Ages in US Adults. International Journal of Biomedical Computing, 39, 209-217. http://dx.doi.org/10.1016/0020-7101(94)01068-C

Chung, S. J. (2007). Computer-Assisted Predictive Formulas Expressing Survival Probability and Life Expectancy in US Adults, Men and Women, 2001. Computer Methods and Programs in Biomedicine, 86, 197-209.

http://dx.doi.org/10.1016/j.cmpb.2007.02.009

Chung, S. J. (2009). Seeking a New World: A New Philosophy of Confucius and Kim Hang. Bloomington, IN: iUniverse.

Chung, S. J. (2010). The Book of Right Change, Jeong Yeok 正易: A New Philosophy of Asia. Bloomington, IN: iUniverse.

Chung, S. J. (2012). The Science of Self, Mind and Body. Open Journal of Philosophy, 2, 171-178. http://dx.doi.org/10.4236/ojpp.2012.23026

Chung, S. J. (2013a). Economic Relationship among Self, Society and Nation. International Journal of Asian Social Science, 3, 622-644. http://www.aessweb.com/pdf-files/622-644.pdf

Chung, S. J. (2013b). Mathematical Relationship of “Probacent”-Probability Equation among Exogenous Stressor, Stress and Response in Biological Phenomena. International Journal of Education and Research, 1, 1-32. 
http://www.ijern.com/journal/September-2013/01.pdf

Chung, S. J. (2014). Self and Consciousness: Mind and Matter. International Journal of Education and Research, 2, 1-28. http://www.ijern.com/journal/March-2014/35.pdf

Close, F. (2011). The Infinity Puzzle. New York: Basic Books.

Dalai Lama (2005). The Universe in a Single Atom (pp. 35-38). New York: Morgan Road Books.

Darling, D. (2005). Teleportation: The Impossible Leap. Hoboken, NJ: John Wiley \& Sons.

Dirac, P. (2014). Dirac Equation. http://en.wikipedia.org/wiki/Dirac.equation

Greene, J. R., Sommerville, R. B., Nystrom, L. E., Darley, J. M., \& Cohen, J. D. (2001). An fMRI Investigation of Emotional Regulation in Moral Judgment. Science, 293, 2150-2108. http://dx.doi.org/10.1126/science.1062872

Greyson, B. (2011). Cosmological Implications of Nrea-Death Experiences. In Consciousness and the Universe (pp. 389402). Cambridge, MA: Cosmology Science Publishers.

Gruenberg, L. A. (1959). Defining Moments: The Korean War. Detroit, MI: Omnigraphics.

Griffiths, D. J. (2005). Introduction to Quantum Mechanics. Noida: Pearson.

Hameroff, S. (2013). Quantum Computation in Brain Microtubules? The Hameroff 'Orch OR' Model of Consciousness. Philosophical Transactions of the Royal Society of London, A (1998), 356, 1869-1896.

Hawking, S. (1996). A Brief History of Time. New York: Bantam Books.

Hawking, S. (2001). The Illusrated a Brief History of Time. New York: Bantam Books.

Herman, S. P., Ostlander, M. M., Mueller, N. K., \& Figueiredo, H. (2005). Limbic System Mechanism: Hypothalamus-Pituitary-Adrenal Axis. Progress in Nuro-Psychopharmacology and Biological Psychiatry, 29, 1201-1213.

Joseph, R. (2001). The Limbic System and the Soul: Evolution and the Neuroanatomy of the Religious Experience. Zygon: The Journal of Religion and Science, 36, 105-136. http://dx.doi.org/10.1111/0591-2385.00343

Joseph, R. (2011a). Dreams and Hallucinations: Lifting Veil to Multiple Perceptual Realities. In R. Penrose, S. Hameroff, \& S. Kak (Eds.), Consciousness and the Universe (pp. 516-550). Cambridge, MA: Cosmology Science Publishers.

Joseph, R. (2011b). Evolution of Paleolithic Cosmology and Spiritual Consciousness, and the Temporal and Frontal Lobes, In R. Penrose, S. Hameroff, \& S. Kak (Eds.), Consciousness and the Universe (pp. 631-382). Cambridge, MA: Cosmology Science Publishers.

Joseph, R. (2011c). The Split Brain: Two Brain-Two Minds. In Consciousness and the Universe (pp. 108-152). Cambridge, MA: Cosmology Science Publishers.

Joseph, R. (2011d). Quantum Physics and the Multiplicity of Mind: Split-Brains, Fragmented Minds, Dissociation, Quantum Consciousness. In Consciousness and the Universe (pp. 976-1007). Cambridge, MA: Cosmology Science Publishers.

Kaku, M. (1999). Introduction to Superstrings and M-Theory (2nd ed.). New York: Springer.

Kim, H. (1885). Jeong Yeok, 金恒, 正易, 정역. The Original Chinese Text with the Text Translated in Korean by Jeong Ho Yi (1990). Seoul: The Asian Culture Press.

Laszlo, E. (2007). Science and the Akashic Field. Rochester, VT: Inner Traditions.

Laszlo, E. (2008). Quantum Shift in the Global Brain. Rochester, VT: Inner Traditions.

McGonigal, K. (2012). The Willpower Instinct. New York: Avery.

Miller, E. K., \& Cohen, J. D. (2001). An Integrative Theory of Prefrontal Cortex Function. Annual Review of Neuroscience, 24, 167-202. http://dx.doi.org/10.1146/annurev.neuro.24.1.167

Mitchell, E. D., \& Staretz, R. (2011). The Quantum Hologram and the Nature of Consciousness. In R. Penrose, S. Hameroff, \& S. Kak (Eds.), Consciousness and the Universe (pp. 933-965). Cambridge, MA: Cosmology Science Publishers.

National Geography Society (2008). 1000 Events that Shaped the World. Washington DC: National Geography Society, 18-19.

Nichol, L. (2006). The Essential David Bohm. New York: Routledge.

Ochsner, K. N., Bunge, S. A., Gross, J. J., \& Gabrieli, J. D. (2002). Rethinking Feelings: An fMRI Study of the Cognitive Regulation of Emotion. Journal of Cognitive Neuroscience, 14, 1215-1229.

Penrose, R. (2007). The Road to Reality: A Complete Guide to the Laws of the Universe. New York: Vintage Books.

Penrose, R., \& Hameroff, S. (2011). Consciousness in the Universe: Neuroscience, Quantum Space-Time Geometry and Orch OR Theory. In Consciousness and the Universe: Quantum Physics, Evolution, Brain and Mind (pp. 3-42). Cambridge, MA: Cosmology Science Publishers.

Penrose, R., Hameroff, S., \& Kak, S. (2011). Consciousness and the Universe. Cambridge, MA: Cosmology Science Pub- 
lishers.

Radin, D. (1997). The Conscious Universe: The Scientific Truth of Psychic Phenomena. San Francisco, CA: Harper.

Rational Buddhism (2012). Buddhism, Quantum Physics and Mind. http://rational-buddhism.blogspot.com/2012/buddhism-quantum

Rilling, J. K., \& Insel, T. R. (1999). The Primate Neocortex in Comparative Perspective Using Magnetic Resonance Imaging. Journal of Human Evolution, 37, 191-223. http://dx.doi.org/10.1006/jhev.1999.0313

Schwartz, J. M. (1999). A Role for Volition and Attention in the Generation of New Brain Circuitry: Toward a Neurobiology of Mental Force. Journal of Consciousness Study, 6, 115-142.

Schwartz, J., Stapp, H. P., \& Beaureguard, M. (2005). Quantum Physics in Neuroscience and Psychology: A Neurophysical Model of Mind-Brain Interaction. Philosophical Transactions of the Royal Society: B, 360, 1309-1327. http://dx.doi.org/10.1098/rstb.2004.1598

Schultz, W., Trembler, L., \& Hollerman, J. R. (2000). Reward Processing in Primate Orbitofrontal Cortex and Basal Ganglia. Cerebral Cortex, 10, 272-283. http://dx.doi.org/10.1093/cercor/10.3.272

Smith, A. R. (2008). Nucleic Acids to Amino Acids: DNA Specifies Protein. Nature Education, 1, 126. http://www.nature.com/scitable/topicpage/nucleic-acids-to-amino-acids-dna-specifies-935

Stapp, H. P. (1999). Attention, Intention, and Will in Quantum Physics. Journal of Consciousness Studies, 6, 143-164.

Stapp, H. (2011). Mindful Universe: Quantum Mechanics and the Participating Observer. New York: Springer.

Suplee, C. (1999). Physics in 20th Century. New York: Harry N. Abrams, Inc.

Tong, D. (2009). String Theory. http://www.damtp.cam.ac.uk/user/tong/string/string.pdf

Vedral, V. (2010). Decoding Reality. New York: Oxford University Press.

Watson, A. (1997). Quantum Spookiness Wins, Einstein Loses in Photon Test. Science, 277, 481.

Wikipedia (2012). Philosophy of Self. http://en.wikipedia.org/wiki/Philosophy_of_self

Wikipedia (2013a). Can Quantum Effects Explain Consciousness? http://thebrain.mcgill.ca/flash/a/a_12/a_12_m/a_12_m_con/a_12_m_con.html

Wikipedia (2013b). Electron Shell. http://en.wikipedia.org/wiki/Electron_shell

Wikipedia (2014). Genetic Code. http://en.wikpedia.org/w/index.php?title_Genetic_code.oldid=589321240

William, R. (1967). The I Ching or the Book of Changes. Rendered into English by C. F. Baynes. London: Rutledge \& Kegan Paul.

Yi, J. H. (1992). The Third Yeok Hak, 第三의易學 (The Author’s Note: Jeong Yeok as the Third Yeok) (Text in Korean). Seoul: The Asian Culture Press. 